\title{
Review of Alpha-1-Antitrypsin Deficiency Associated Panniculitis
}

\author{
Anand Rajpara ${ }^{*}$, Corinne Erickson and Marcia Driscoll
}

Department of Dermatology, University of Maryland School of Medicine, Baltimore, MD, USA

\begin{abstract}
Alpha-1-Antitrypsin (AAT) deficiency associated panniculitis is commonly included in the dermatologist's differential diagnosis but the disease is rarely ever diagnosed or discussed. The pathophysiology, clinical presentation, histopathology, diagnosis, and treatment of AAT deficiency panniculitis will be reviewed.
\end{abstract}

Keywords: AAT panniculitis, panniculitis, A1AT panniculitis.

\section{INTRODUCTION}

Alpha1-Antitrypsin (AAT) deficiency is defined by a reduced level of AAT concentration in the serum and/or the identification of a defective protein phenotype or genotype. It is an autosomal co-dominant genetic disease that affects about one in 3000-5000 individuals in the United States [1]. AAT deficiency appears to be an under-diagnosed disease. In the United States it is estimated that 60,000 patients have symptomatic AAT deficiency but less than 10,000 have actually been identified. The main pathological consequence of AAT deficiency is panacinar emphysema, but liver cirrhosis, noninfectious hepatitis, and persistent vasculitis are also known to occur in AAT deficient patients [2]. AAT deficiency associated panniculitis was first described by Warter et al. [3] in 1972, and since then, over 40 cases have been reported in the literature. AAT deficiency panniculitis will be reviewed here.

\section{NORMAL AAT PROTEIN}

AAT is a member of the serine protease inhibitor (serpin) superfamily of proteins. AAT is mainly produced and secreted by the liver and is the most abundant circulating serpin in humans. The protein plays a critical role in maintaining protease-antiprotease balance by inhibiting several serine proteases, most importantly of which is neutrophil elastase. In addition to its anti-protease activity, AAT also plays a role in the down regulation of inflammatory processes by blocking the pro-inflammatory effects of human neutrophil peptide and decreasing expression of pro-inflammatory cytokines [1]. In addition to being constitutively expressed, AAT is an acute phase reactant and is upregulated during inflammation, cancer, infection, and pregnancy [4].

\section{AAT GENETIC ALLELES}

AAT is encoded by the highly pleomorphic SERPINA1 gene located on chromosome 14. To date there are over 100 genetic variants of the AAT allele, which can be classified into 4 main categories: normal alleles, dysfunctional alleles,

*Address correspondence to this author at the Department of Dermatology, University of Maryland School of Medicine, Baltimore, MD, USA; Tel: 410-328-5767; E-mail: arajpara@gmail.com deficient alleles, and null alleles. Normal alleles produce functional protein and normal serum concentration of AAT $(80-220 \mathrm{mg} / \mathrm{dl})$. Deficient alleles produce a defective protein that polymerizes within hepatocytes and/or is degraded intracellulary, leading to decreased liver secretion of AAT and low serum levels $(<80 \mathrm{mg} / \mathrm{dl})$. Null alleles synthesize no protein and thus produce undetectable levels of serum AAT. Finally, dysfunctional alleles code for proteins with decreased serpin function and may or may not produce normal AAT serum concentrations. Alleles can both be dysfunctional and deficient at the same time $[1,4]$. AAT deficiency/dysfunction leads to a protease-antiprotease imbalance that favors tissue proteolysis, which is thought to be the cause of both panacinar emphysema and panniculitis. The associated cirrhosis and hepatitis seen in AAT deficiency are thought to be due to the accumulation of AAT polymers within hepatocytes.

\section{AAT PROTEIN PHENOTYPES}

Clinically, AAT protein variants are phenotyped into 4 main categories based on their mobility on acid-starch electrophoresis gel: Z-very slow, S-slow, M-medium, and Ffast. The M proteins are the most common and are defined as normal proteins as they result in normal levels of AAT, and MM homozygotes have no associated disease. Conversely, Z proteins are the most common pathogenic mutants. The most common Z protein contains a lysine for glutamate substitution at position 342 and accounts for $95 \%$ of clinically recognized cases of AAT deficiency. This mutation causes the AAT protein to polymerize and accumulate within hepatocytes, leading to secretion of only $15 \%$ of the formed $Z$ protein. Further, the $Z$ protein that is secreted is 5 times less effective at inhibiting neutrophil elastase than normal AAT. The $\mathrm{S}$ proteins are also pathogenic mutants but are less common and cause less severe disease than the $\mathrm{Z}$ proteins. The most common $\mathrm{S}$ protein has a valine for glutamate substitution at position 264, which leads to intra-hepatic degradation and polymerization, but to a lesser extent than the $\mathrm{Z}$ protein. Most cases of AAT-deficiency panniculitis have been reported in patients with a $\mathrm{ZZ}$ protein phenotype and less commonly in patients with MZ, MS, SZ, and SS phenotypes $[1,5]$. 


\section{V.PATHOGENESIS}

The pathogenesis of AAT deficiency panniculitis is not clearly understood. It is generally agreed upon that one of the key mechanisms in this disease is the overactivity of membrane bound serine proteases and neutrophil elastases in the subcutaneous fat due to AAT deficiency and/or dysfunction [2]. Recently, Gross et al. [6] demonstrated Ztype AAT polymers in the skin of a patient with AAT panniculitis and proposed that the AAT polymers, in addition to being non-functional inhibitors of proteases, were actually now pro-inflammatory molecules [6]. Nevertheless, it is clear that AAT deficiency panniculitis is a multifactorial disease with AAT deficiency being just one important factor, considering that this condition only effects 1 in 1000 AAT deficient patients [1].

\section{CLINICAL PRESENTATION}

In 2004 Geraminejad et al. [5] reviewed all 43 reported cases of AAT deficiency in the literature and found that the disease had consistent clinical features. The majority of patients were female $(65.1 \%)$ and the age of patients ranged from 7 to 73 with most cases presenting between the ages of 30 and 60. Most patients presented with recurrent erythematous plaques and painful nodules on the proximal extremities and buttocks that eventually ulcerated and expressed an oily fluid. Importantly, the ulceration and oily discharge characteristically seen in AAT deficiency panniculitis helps to distinguish it from other forms of panniculitis. Further, $35 \%$ of patients reported trauma or excessive activity involving affected areas of the body [5].

\section{HISTOPATHOLOGY}

The histopathology of AAT deficiency panniculitis is characterized into 3 distinct phases which correlate with the 3 stages of clinical lesions. A biopsy of erythematous plaques or nodules typically reveals a neutrophilic infiltrate in the reticular dermis with characteristic splaying between the collagen bundles. The subcutaneous fat typically contains a lobular neutrophilic infiltrate, but mixed lobular and septal patterns of inflammation can also be seen. A biopsy of ulcerated lesions typically reveals liquefactive necrosis of the subcutaneous fat with dissolution of dermal collagen. Finally, biopsy of end-stage, clinically scarred lesions usually reveals scarring and fibrosis with minimal to no inflammation [7]. A clinical photo of a patient with AAT deficiency panniculitis resulting in an erythematous, ulcerated plaque of the proximal thigh is shown in Fig. (1). Low-power and high-power histological images corresponding to the lesion shown in Fig. (1) are depicted in Fig. (2).

\section{DIAGNOSIS}

The differential diagnosis of AAT deficiency includes infectious panniculitis, pancreatic panniculitis, and foreign body panniculitis [8]. All of these panniculitides have a lobular pattern of inflammation of the subcutaneous fat with a predominantly neutrophilic inflammatory infiltrate and no evidence of vasculitis [8]. Key distinguishing histological features of panniculitides in the differential diagnosis of AAT deficiency panniculitis are presented in Table 1 [8]. The diagnosis of AAT deficiency panniculitis should be considered in any patient with ulcerative or necrotizing panniculitis with oily drainage. The initial step in diagnosis consists of sending a deep wedge biopsy for histopathology. If the histopathology is consistent with AAT deficiency panniculitis, then AAT serum concentrations and an AAT protein phenotype should be obtained. AAT serum concentrations alone are insufficient because $25 \%$ of reported cases of AAT deficiency panniculitis had normal serum concentrations of AAT [5]. Ultimately, the diagnosis of AAT deficiency panniculitis can be made based on a decreased serum concentration of AAT and/or abnormal protein phenotype in the setting of classic clinical and histological features [5].

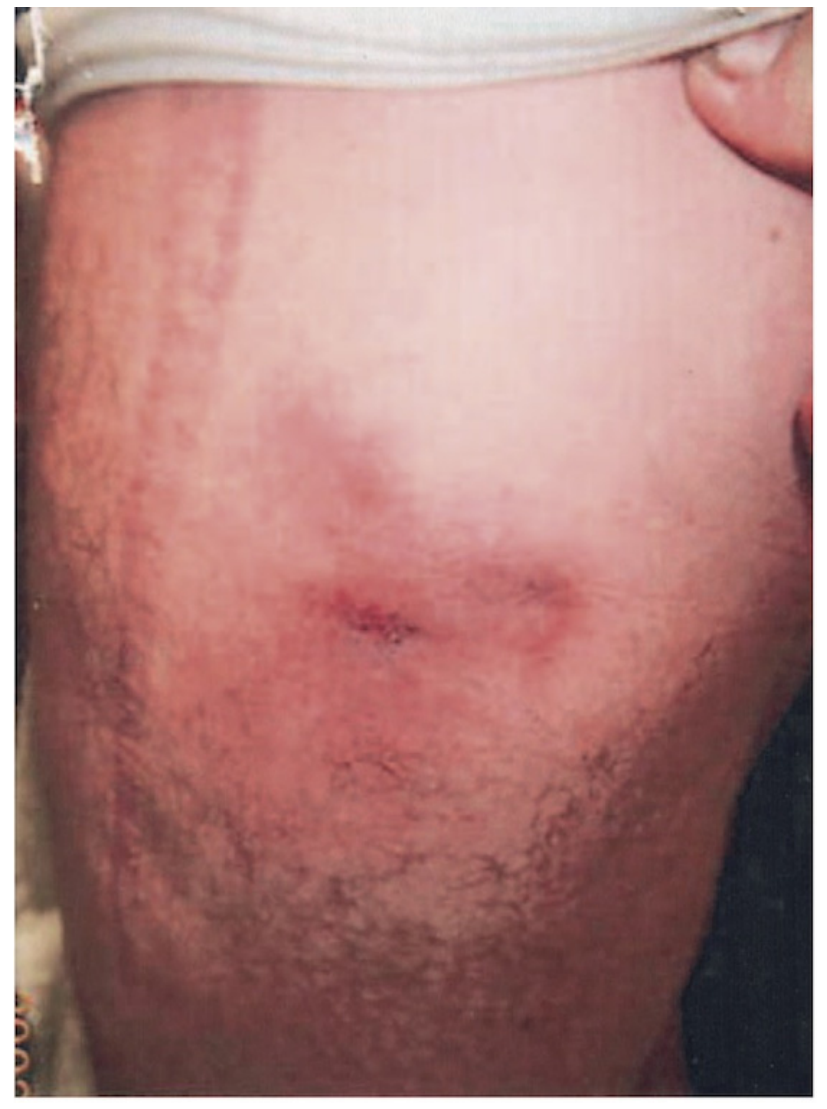

Fig. (1). Patient with AAT deficiency panniculitis resulting in an erythematous, ulcerated plaque of the proximal thigh (reprinted from Journal of the American Academy of Dermatology, Volume 51, Geraminejad P, DeBloom JR, Walling HW, Sontheimer RD, VanBeek M, Alpha-1-antitrypsin associated panniculitis: the MS variant, Pages 645-655, Copyright 2004 with permission from Elsevier).

Table 1. Key Distinguishing Histological Features of Panniculitides in the Differential Diagnosis of AAT Deficiency Panniculitis

\begin{tabular}{|c|c|}
\hline Type of Panniculitis & Key Histological Features \\
\hline \hline $\begin{array}{c}\text { AAT deficiency } \\
\text { panniculitis }\end{array}$ & $\begin{array}{c}\text { Neutrophils between collagen } \\
\text { bundles in the deep reticular dermis }\end{array}$ \\
\hline Pancreatic panniculitis & $\begin{array}{c}\text { Extensive fat necrosis with } \\
\text { saponification of adipocytes }\end{array}$ \\
\hline Infectious panniculitis & Evidence of bacteria, fungi, or protozoa \\
\hline Factitial panniculitis & Evidence of foreign bodies \\
\hline
\end{tabular}



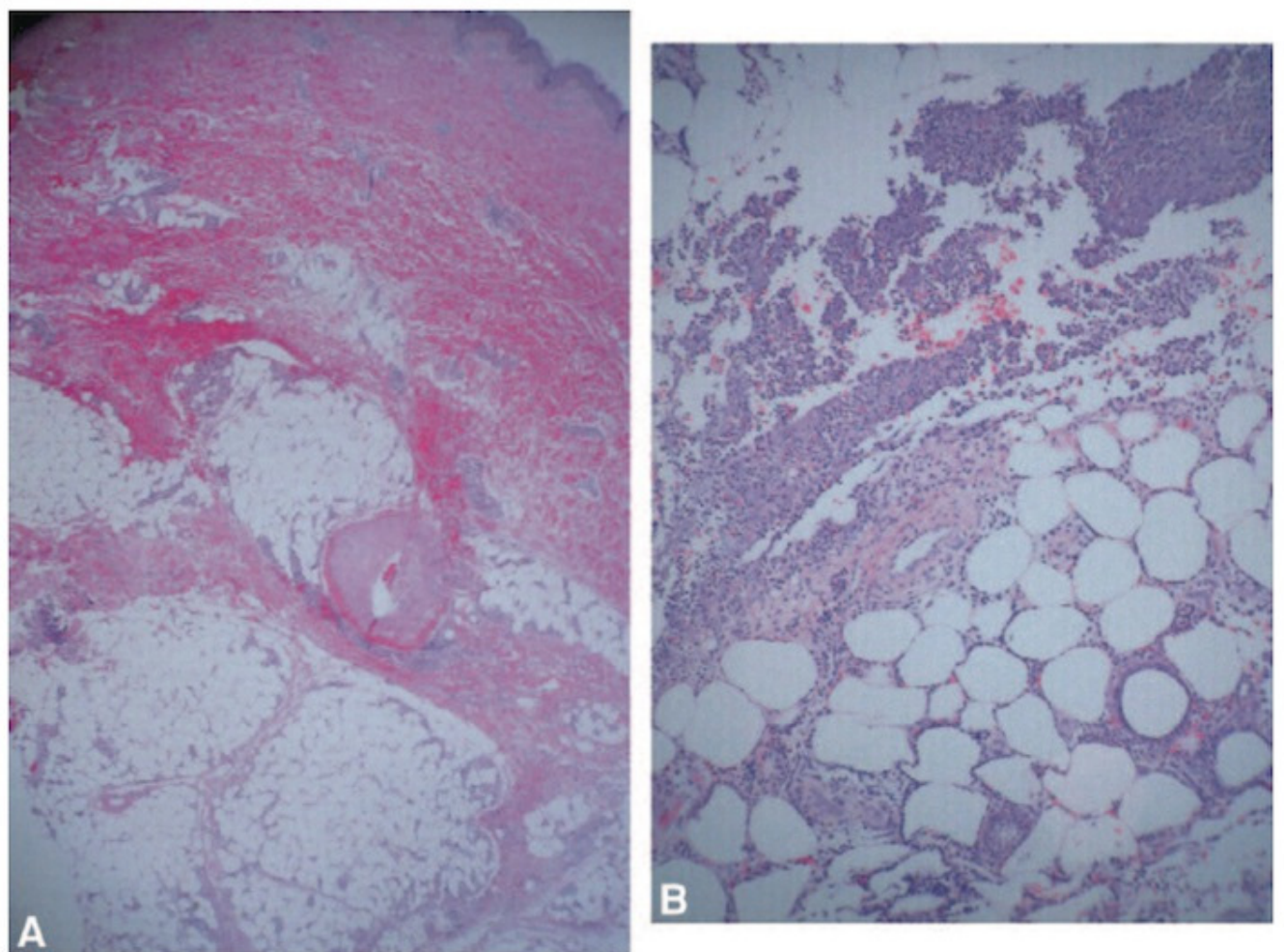

Fig. (2). Low-power and high-power histological images of AAT deficiency panniculitis corresponding to the lesion shown in Fig. (1) (Reprinted from Journal of the American Academy of Dermatology, Volume 51, Geraminejad P, DeBloom JR, Walling HW, Sontheimer RD, VanBeek M, Alpha-1-antitrypsin associated panniculitis: the MS variant, Pages 645-655, Copyright 2004 with permission from Elsevier).

\section{TREATMENT}

As there are less than 50 reported cases of AAT deficiency panniculitis, there are no standardized guidelines for treatment, and numerous therapeutic agents have been tried with varying success [6]. Most treatments are aimed at correcting the protease-antiprotease imbalance that is believed to be a key mechanism in the disease process. Oral dapsone at a dose of $50-150 \mathrm{mg}$ daily is considered to be a first-line therapeutic agent for treating AAT panniculitis because it has been the most common agent used and it is effective. As of 2009, treatment of AAT panniculitis with oral dapsone has been documented in the literature in 23 cases. Of those 23 cases, oral dapsone improved AAT deficiency panniculitis in 21 cases. AAT replacement seems to be a highly effective therapy for treating AAT panniculitis and has been documented to rapidly improve disease in 3 out of 3 cases as of 2009. However, AAT replacement is extremely expensive and not FDA approved for panniculitis. Accordingly, AAT replacement therapy is considered only in those patients with life-threatening panniculitis or concomitant lung or liver disease. The tetracyclines, through their anti-collagenase and anti-lipase activities, have been documented to effectively treat AAT deficiency panniculitis in 7 out of 8 cases in the literature since 2009. Thus, tetracyclines at doses around $200 \mathrm{mg}$ per day can serve as an attractive therapeutic option for those patients who cannot tolerate oral dapsone [6].

\section{OTHER CONSIDERATIONS}

After diagnosing a patient with AAT deficiency, it is important that the dermatologist council the patient on his/her risk of lung and liver disease, which are far more common clinical manifestations of AAT deficiency than panniculitis. The main pathological consequence of AAT deficiency is bilateral lower lobe panacinar emphysema, which results from the unopposed activity of neutrophil elastase on lung tissue. AAT deficiency can also cause liver cirrhosis and hepatitis which results from the build up of mutant AAT polymers within hepatocytes. Accordingly, dermatologists should refer AAT deficient patients to the pulmonologist or gastroenterologist if there is any suspicion of lung or liver involvement [9].

\section{REFERENCES}

[1] Stoller JK, Aboussouan LS. Alpha-1 antitrypsin deficiency. Lancet 2005; 365: 2225-36.

[2] Ortiz PG, Skov BG, Benfeldt E. Alpha1-antitrypsin deficiencyassociated panniculitis: case report and review of treatment options. J Eur Acad Dermatol Venereol 2005; 19: 487- 90.

[3] Warter J, Storck D, Grosshans E, Metais P, Kuntz JL, Klumpp T. Weber-Christian syndrome associated with an alpha-1 antitrypsin deficiency. Familial investigation. Ann Med Interne (Paris) 1972; 123: 877-82.

[4] Köhnlein T, Welte T. Alpha-1 antitrypsin deficiency: pathogenesis, clinical presentation, diagnosis, and treatment. Am J Med 2008; 121:3-9

[5] Geraminejad P, DeBloom JR, Walling HW, Sontheimer RD, VanBeek M. Alpha-1-antitrypsin associated panniculitis: the MS variant. J Am Acad Dermatol 2004; 51: 645-55. 
[6] Gross B, Grebe M, Wencker M, Stoller JK, Bjursten LM, Janciauskiene S. New findings in PiZZ alphal-antitrypsin deficiencyrelated panniculitis. Demonstration of skin polymers and high dosing requirements of intravenous augmentation therapy. Dermatology 2009; 218: 370-5.

[7] Piliang M, Stoller J. The clinical picture: a woman with ulcerating painful skin lesions. Cleveland Clin J Med 2008; 75: 414-22.
[8] Segura S, Requena L. Anatomy and histology of normal subcutaneous fat, necrosis of adipocytes, and classification of the panniculitides. Dermatol Clin 2008; 26: 419-24.

[9] Silverman EK, Sandhaus RA. Clinical practice: alpha1-antitrypsin deficiency. N Engl J Med 2009; 360: 2749-57.

Received: April 12, 2010

(C) Rajpara et al.; Licensee Bentham Open.

This is an open access article licensed under the terms of the Creative Commons Attribution Non-Commercial License (http://creativecommons.org/licenses/by-nc/ 3.0/) which permits unrestricted, non-commercial use, distribution and reproduction in any medium, provided the work is properly cited. 